\title{
Cross-correlation analysis of synchronized PIV and \\ microphone measurements of an oscillating airfoil
}

\author{
Lars Siegel · Klaus Ehrenfried · Claus
}

Wagner · Karen Mulleners • Arne Henning

Received: date / Accepted: date

\begin{abstract}
The present study focusses on the correlation between the flow structures evolving during the dynamic stall processes of a two-dimensional periodically pitching NACA64-618 airfoil and their aeroacoustic response in the far field. Experiments are conducted in an anechoic wind tunnel at a Reynolds number of $8 \times 10^{5}$ based on the chord length and include simultaneous velocity field measure-

L. Siegel · C. Wagner

German Aerospace Center (DLR), Institute for Aerodynamics and Flow Technology, Göttingen, Germany. Tel.: +49 (0)551 7092269 E-mail: lars.siegel@dlr.de

Second institute: Technische Universität Ilmenau, Institute of Thermodynamics and Fluid Mechanics, Ilmenau, Germany.
\end{abstract}

Klaus Ehrenfried · A. Henning

German Aerospace Center (DLR), Institute of Aerodynamics and Flow Technology (AS), Göttingen, Germany

K. Mulleners

École Polytechnique Fédérale de Lausanne (EPFL), UNFOLD, Lausanne, Switzerland 
ments in the vicinity of the airfoil and microphone measurements in the acoustic far field. A causality correlation method based on phase locked snapshots of the velocity field allows for the identification of specific structures at different phases of the dynamic stall life cycle that contribute to the sound generation process. The sound emission during the stall development and flow reattachment phases is attributed to coherent structures evolving downstream of the trailing edge. Further, when the flow is fully stalled, the region that contributes to the sound emission increases. The reason is that the locations of the sound emitting coherent structures fluctuate stronger between oscillation cycles.

Keywords PIV, aero-acoustics · dynamic stall · cross-correlation · coherent structures

\section{Introduction}

If the angle of attack of an airfoil exceeds a certain value the flow can detach and the airfoil stalls, which is associated with a significant drop in lift. This critical angle is referred to as the static stall angle of attack. Dynamic pitching oscillations up to angles of attack beyond this static limit can lead to periodic flow detachment and reattachment or dynamic stall. Dynamic stall is associated with the generation and shedding of large-scale dynamic stall vortices and a delayed onset of the flow separation with respect to static stall (Carr, 1988; McAlister et al, 1978; McCroskey, 1981). The flow development during dynamic stall has been divided into different stages (Carr et al, 1977; Doligalski et al, 1994; Mulleners and Raffel, 2013). Based on velocity field measurement using particle image velocimetry (PIV) additional details of the dynamic stall development were identified and lead 
to the introduction of the concept of the dynamic stall life cycle (Mulleners and Raffel, 2013; Raffel et al, 1995; Zanotti et al, 2014). The cycle starts for moderate angles of attack with the attached flow stage. With increasing angle of attack, flow reversal emerges on the suction side of the airfoil. Depending on the airfoil shape, stall development further manifests itself by either a upstream motion of the trailing edge separation region or shear layer roll-up (Degani et al, 1998; Gupta and Ansell, 2017; Reynolds and Carr, 1992). The shedding of the primary dynamic stall vortex marks stall onset and the start of the full stalled stage (Mulleners and Raffel, 2012; Obabko and Cassel, 2002). During the downstroke of the airfoil, the flow remains fully separated until lower angles of attack are reached and the flow reattaches.

The role of the characteristic flow features that are associated with dynamic stall on the dynamic loads and especially on the noise emission is not yet fully understood and remains an import research topic in the field of helicopter and wind turbine aerodynamics. For wind turbines, the pitching of the rotor blades and the associated dynamic stall phenomenon is an important control mechanism for load reduction (Bossanyi, 2003; Petrovic, 2008). Hitherto, most studies have focused on the aerodynamic or aero-elastic effects of this process. The aero-acoustic effect of dynamic stall has not been explored with the same efforts. Due to the changing flow characteristics during the pitching motion, different noise mechanisms occur including fluid-structure interactions as well as near- and far-field impacts (Manela, 2013). Trailing edge noise is considered to be the dominant noise source of wind turbines (Brooks and Humphreys Jr., 2003; Oerlemans and Migliore, 2004; Wolf et al, 2014). When the blade trailing edge moves as a result of an active flap or blade pitch motion, the noise source will move along a complex 
trajectory and interact with the moving blades trailing edge (Ffowcs Williams and Hawkings, 1969). This complicates not only the localization and characterization of the source of the noise, but also the sound emission characteristics. Various concepts and prediction models have been already developed in order to reduce the noise emission since noise constraints have to be maintained for wind turbines in proximity to urban regions (Doolan et al, 2012; Nagarajan et al, 2006; Wolf et al, 2014). In this paper, the flow structures associated with the various dynamic stall development stages will be compared to the airfoil self-noise mechanisms (Brooks et al, 1989), which is predominantly the vortex-shedding noise at the trailing edge and the separation-stall noise due to large-scale separation during the deep stall process. Two-dimensional three-component (2D3C) stereoscopic particle image velocimetry is conducted in the near-field of an oscillating airfoil simultaneously with microphone measurements in the far-field, which provide the acoustic pressure fluctuations. This synchronized aero-acoustic measurement technique was successfully applied in the past to fixed bodies like e.g. a rod-airfoil configuration (Henning et al, 2010) or a high-lift device (Henning et al, 2012). These studies revealed that this approach provides the link between coherent flow structures and aero-acoustic noise mechanisms. In continuation of the previous work, the application range of the measurement technique is extended to the oscillating airfoil case to explore its limitations and its applicability and to study the aero-acoustic footprint of dynamic stall. 
2 Theoretical background

Based on the measured velocity and pressure fluctuations, the cross-correlation function $S_{\phi, p}$ and coefficients $R_{\phi, p}$ are calculated in order to identify flow structures that are statistically correlated to the aero-acoustic source mechanisms (Henning et al, 2010). The calculation scheme of the cross-correlation operation is presented in figure 1 and the mathematical definition of these quantities is given by:

$$
S_{\phi, p}(\mathbf{x}, \tau)=\frac{1}{N} \sum_{i=1}^{N}\left(\phi\left(\mathbf{x}, t_{i}\right)-\bar{\phi}(x)\right) \cdot p^{\prime}\left(t_{i}-\tau\right) .
$$

Here, $\phi$ symbolizes the near-field quantity e.g. a velocity component of the flow field obtained with PIV at the positions $\mathbf{x}$ and the points in time $t_{i}$. The spatiotemporal function $\bar{\phi}$ denotes the ensemble averaged value of the near-field quantity and $N$ specifies the overall number of PIV snapshots. The pressure fluctuations recorded with microphones in the far-field are indicated by $p^{\prime}$. As the sampling rate of the acoustic recording is much higher than the PIV recording rate $(100 \mathrm{kHz}$ vs $14 \mathrm{~Hz})$, the variable $\tau$ is introduced representing a time window covering 4097 acoustic samples including 2048 samples before and 2048 samples after the individual PIV snapshots. The time window applies to every single PIV snapshot and defines the temporal resolution of the cross-correlation results as illustrated in figure 1. Normalizing the correlation function with the standard derivations $\sigma_{\phi}(\mathbf{x})$ and $\sigma_{p}(\tau)$ of the velocity and the pressure fluctuations, respectively, yields the correlation coefficients:

$$
R_{\phi, p}(\mathbf{x}, \tau)=\frac{S_{\phi, p}(\mathbf{x}, \tau)}{\sigma_{\phi}(\mathbf{x}) \cdot \sigma_{p}(\tau)}
$$

The calculation of the cross-correlation values can be executed in two different ways. The time between subsequent PIV snapshot (1/14 s) and the oscillation 


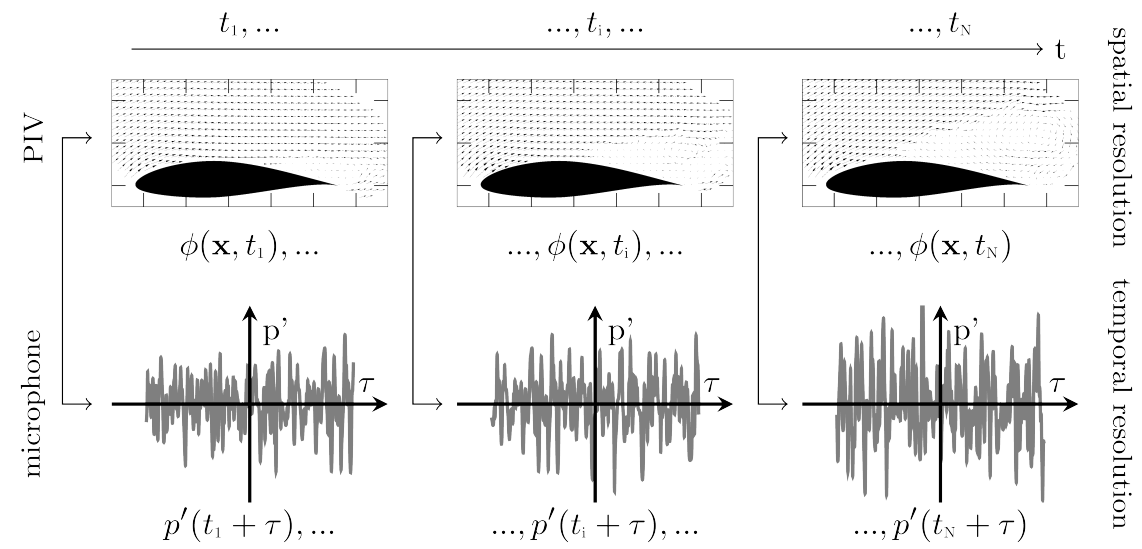

Fig. 1 Calculation scheme of the cross-correlation of the fluctuating velocity fields measured via PIV (top) and the pressure signal recorded with a microphone (bottom).

period of the airfoil pitching motion $(1 / 5 \mathrm{~s})$ have a least common multiple of 1 and every $14^{\text {th }}$ snapshot is recorded at the same phase angle of the pitching motion. Hence, phase locked data for 14 different phase angles are recorded during the full oscillation process. This allows to either consider the full data set or an ensemble of phase-locked snapshots at selected phase angles for cross-correlation analysis. Additionally, the standard deviation of the pressure is now a function of $\tau$ due to the periodic movement of the profile. This is an important difference to the approaches for flows around stationary objects described previously, in which the standard deviation of the far-field pressure fluctuations for the entire process is considered (Henning et al, 2010, 2012). With the aim to study the aero-acoustic footprint of characteristic coherent structures that emerge during different phases of the dynamic stall life cycle, the presented results and discussion are focussed on the results of the ensemble of phase-locked snapshots at selected phase angles. 
3 Experimental set-up and data processing

3.1 Flow configuration

The experiments were conducted in the Aeroacoustic Wind Tunnel Brunswick (AWB) of the DLR, German Aerospace Center. The AWB is an open-jet closedcircuit anechoic test facility with a rectangular $0.8 \mathrm{~m} \times 1.2 \mathrm{~m}$ nozzle exit. The twodimensional airfoil model with a NACA64-618 profile of chord length $c=0.3 \mathrm{~m}$ and $\operatorname{span} s=1.1 \mathrm{~m}$ was integrated in a modular setup enabling a configuration with side-plates to reduce shear and boundary layer effects of the wind tunnel flow. The airfoil was placed in a uniform flow at a free-stream velocity of $U_{\infty}=40 \mathrm{~m} / \mathrm{s}$ $\left(R e=U_{\infty} c / \nu=8 \cdot 10^{5}\right.$ with $\nu$ the kinematic viscosity). An electric servo motor ensured a sinusoidal movement of the airfoil about its quarter chord axis with an mean angle of attack $\alpha_{0}=20^{\circ}$, amplitude $a_{1}= \pm 8^{\circ}$ and oscillation frequency $f=5 \mathrm{~Hz}$ (reduced frequency $k=\pi f c / U_{\infty}=0.12$ ). The instantaneous angle of attack was measured based on a laser triangulation in such a way that the captured PIV recordings could be assigned to the respective angle of attack. Two images of the measurement setup are presented from different viewing angles in figure 2 .

3.2 Velocity field measurements

The velocity field data were acquired using a stereo PIV system which measured three velocity components in a vertical plane at mid-span (figure $3 \mathrm{~b}$ ). Two CMOS cameras (Type: PCO edge 5.5) with a resolution of $2560 \mathrm{px} \times 2160 \mathrm{px}$ were placed in a $90^{\circ}$ angle to each other left and right downstream of the field of view to record the illuminated particles on the suction side of the airfoil. Scheimpflug adapters 

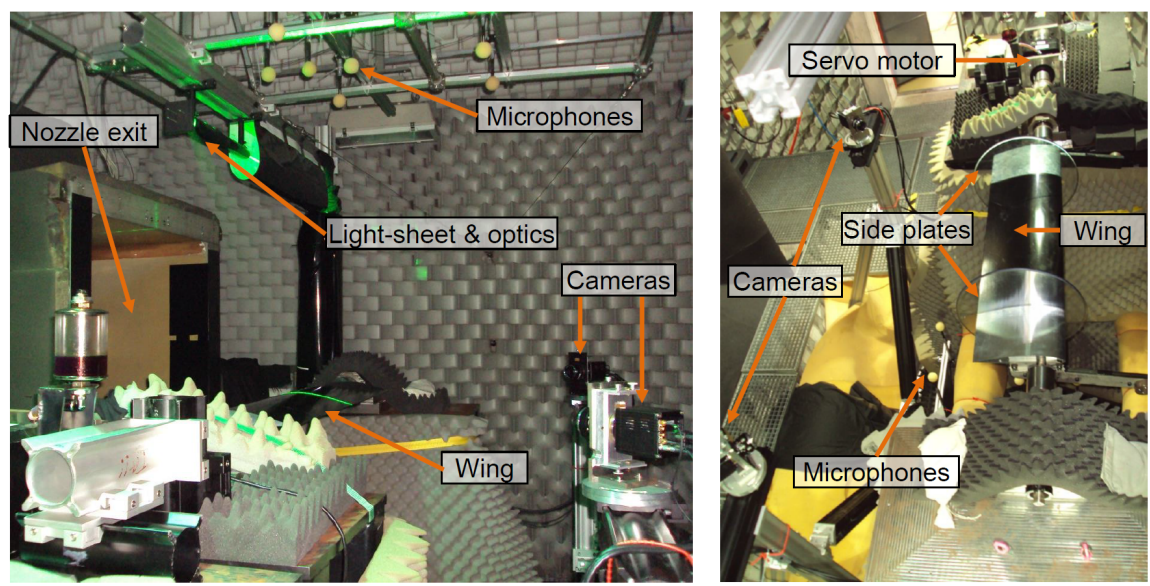

Fig. 2 Images of the experimental setup from two different viewing angles: (left) microphone array above the airfoil, two cameras downstream and light-sheet from inclined above; (right) microphone array below the airfoil (with side plates), two cameras downstream and the servo motor.

were used in order to align the focal- and image-planes therewith compensating for the inclination between the optical axes and the field of view. The recording frequency of the PIV system was $14 \mathrm{~Hz}$ and a total number of 15000 images were recorded in direct-to-disc storage mode for each configuration. The flow was seeded with diethylhexylsebacate (DEHS) tracer particles with a mean particle diameter of approximately $1 \mu \mathrm{m}$ (Raffel et al, 2007). The seeding particles were injected from a corner of the wind tunnel upstream of the model configuration in a way that the particles had to pass the complete wind tunnel before they reached the field of view. The DEHS particles were illuminated using a double-pulse laser (Q-switched Nd:YAG; Type: Innolas Spitlight 600) with a maximum energy of $350 \mathrm{~mJ}$ per pulse and a repetition rate of $14 \mathrm{~Hz}$. The PIV data were recorded simultaneously with the microphone data. The trigger signals for the camera exposure and the laserlight emission were recorded as well in order to be able to subsequently assign the 
corresponding acoustic data to the respective PIV frames. In order to minimize reflections and unwanted scattered light, light absorbing tubes were installed along the beam guidance and the model was equipped with a thin matt black foil.

\subsection{Far-field microphone measurements}

The pressure measurements were conducted with overall 16 microphones (Type: 1/4" 40BF; G.R.A.S.) in the far field outside the flow to avoid unwanted influences on the flow field and vice versa. Eight microphones were installed above and the other eight below the airfoil arranged in a horizontal plane as illustrated in figure 3a. The vertical positions were approximately $1.14 \mathrm{~m}(3.8 c)$ above and $0.99 \mathrm{~m}$ (3.3c) below the airfoil. A multi-analyzer (Type: Viper; GBM) simultaneously recorded the microphone-signals, the camera trigger, the q-switch of the laser and the laser triangulation signals with a sampling frequency of $f_{s}=100 \mathrm{kHz}$ and a dynamic range of 24 bit. All channels had an anti-aliasing filter at $f_{u}=50 \mathrm{kHz}$. To reduce the influence of low-frequency wind-tunnel noise on the measured signals, a high-pass filter with a cutoff frequency $f_{l}=500 \mathrm{~Hz}$ was used. Additionally, the microphones were protected by wind shields against potential flows in the plenum.

\subsection{PIV data processing}

Prior to the evaluation of the velocity fields, several pre-processing steps have to be performed, including data management, filtering, and masking. The velocity vector fields are calculated with a multi-pass stereo cross-correlation algorithm with a final interrogation window size of $32 \mathrm{px} \times 32 \mathrm{px}$ and an overlap of $50 \%$ yielding a physical resolution of $3.16 \mathrm{~mm}$. All vector calculations are performed using a 


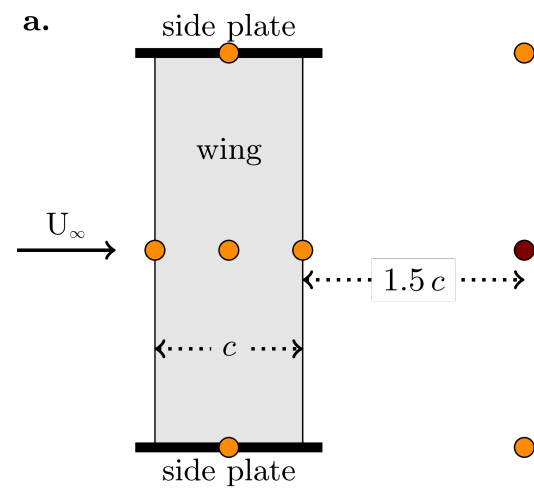

b.

Fig. 3 (a.) Arrangement of the microphones above and below the airfoil in relation to the position of the airfoil and the flow direction (top view). (b.) Schematic of the airfoil, the field of view, and the coordinate system.

high-accuracy mode for the final pass by means of the bi-spline- 6 reconstruction. With these settings, more than 6000 valid velocity vectors were obtained with an average displacement of $10 \mathrm{px}$.

Due to the mentioned phase-locked characteristic of the PIV recording frequency $(14 \mathrm{~Hz})$ and the oscillation frequency of the airfoil $(5 \mathrm{~Hz})$, phase locked data was recorded for 7 phase angles during pitch-up and 7 phase angles during pitch-down. The signal of a single microphone of the upper array is used for the cross-correlation calculation (maroon dot in figure 3a). The following analysis focusses on the values in $y$-direction (the $v$-components of the velocity vectors) which is the direction in which the acoustic perturbations propagate to the microphones. The coordinate system shown in figure $3 \mathrm{~b}$ is used for the representation of the flow field and cross correlation diagrams. The depicted axes are scaled with the chord length of the airfoil while the zero point is set to the pitching axis of the airfoil. All the fields of view are rotated into the airfoil coordinate system. In the flow 
field and cross correlation diagrams presented below, the contour of the profile is drawn in black.

\section{Results and discussion}

4.1 Spectrum and spectrogram of the acoustic signal

Before discussing the flow fields and the cross-correlation results, the acoustic information is presented here. The acoustic spectrum of the selected configuration (black solid line) is compared to those obtained for two reference configurations in figure 4. The maroon line reflects the noise level measured for a non-oscillating airfoil which produces no lift. The orange line displays the acoustic response when the airfoil is completely removed from the setup. All spectra are calculated using 200 individual time series with a length of $2 \cdot 10^{5}$ samples and an overlap of $50 \%$. They are weighted with a Hanning window and Fourier-transformed. The squared values of the resulting short-term spectra are then averaged to represent the sound pressure levels. The threshold of human hearing $\left(p_{0}=2 \cdot 10^{-5} \mathrm{~Pa}\right)$ is used as the reference sound pressure in air. The resulting frequency resolution is $0.38 \mathrm{~Hz}$.

The second acoustic analysis is the generation of a spectrogram from the pressure field of the flow presented in figure 5. The calculation is based on a signal section of $2 \cdot 10^{5}$ samples which represents the general acoustic response regarding to the temporal evolution. Figure 5a displays an excerpt of the full spectrogram for a time period corresponding to one oscillation cycle of the airfoil which represents the acoustic response regarding to the respective phase angle of the airfoil (figure 5c). The selected input parameters, including the window function, the overlap, and the sampling points result in a time resolution of $7 \cdot 10^{-4} \mathrm{~s}$ and a fre- 


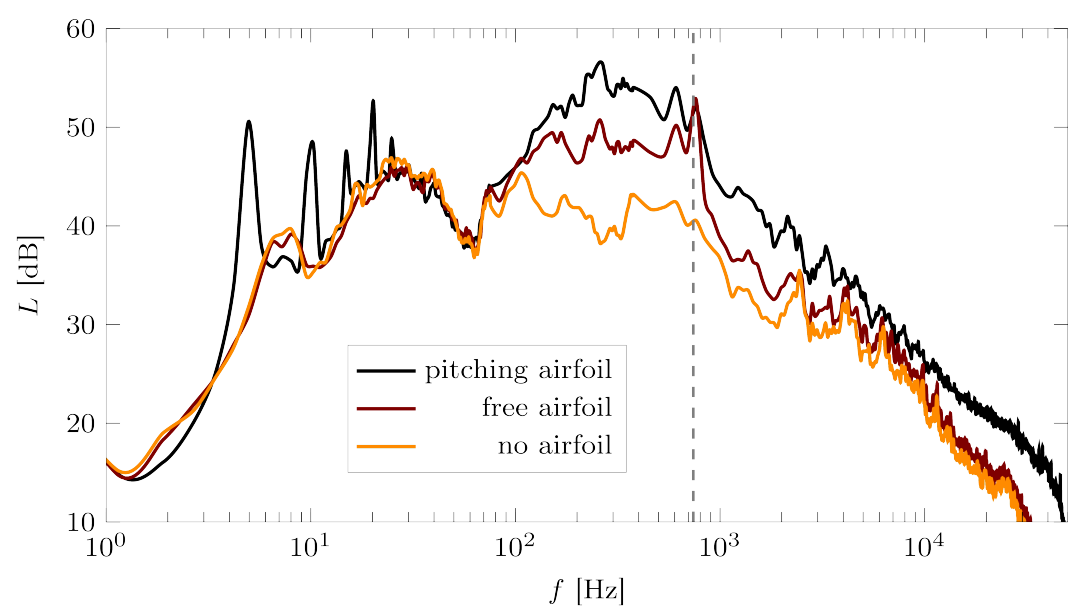

Fig. 4 Spectra of the oscillating airfoil configuration and two reference configurations, a freely hinged airfoil that is not oscillating and not generating lift, and a configuration without the airfoil present.

quency resolution of $12.2 \mathrm{~Hz}$ for the data presented in figure $5 \mathrm{a}$. The spectogram averaged over 500 cycles is shown in figure 5b. Here a short-time broadband signatures can be observed at the time of the PIV recordings, resulting in thin vertical lines in the spectrogram. This typical click sounds coming with the emission of each laser pulse are caused by the discharge of the capacitors providing the energy for the laser flash lamps. It should be noted that they are uncorrelated with the velocity field and therefore, these do not affect the correlation results presented in section 4.3 .

The oscillation frequency of $5 \mathrm{~Hz}$ and its harmonics corresponding to the airfoil motion can be readily identified in the spectrum in figure 4 . This is also reflected in the full spectrogram (not shown here), in which a dominant event takes place every 0.2 seconds. Considering the acoustic response in regard to the oscillation cycle (figure 5a,b), reveals that the overall sound pressure level increases with increasing 

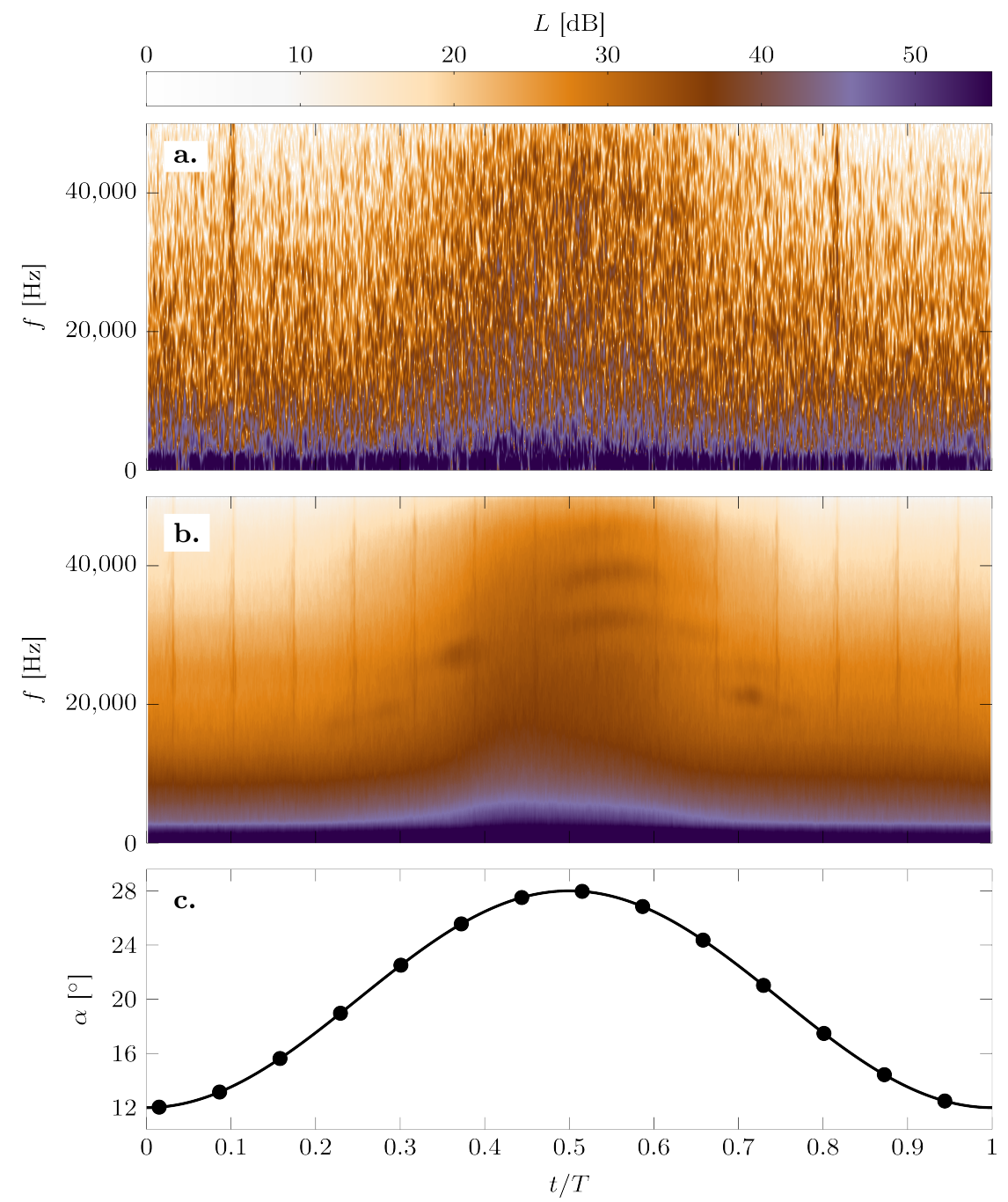

Fig. 5 (a.) Spectrogram of the pitching airfoil configuration for a single oscillation period and (b.) it's phase average over 500 cycles. (c.) The pitching oscillation and the measured phase angles of the selected flow configuration are also depicted in relation to the airfoil oscillation period.

angles of attack $\left(\alpha>20^{\circ}\right)$, especially for higher frequencies. In general, most of the sound energy is contained in the frequencies below $1000 \mathrm{~Hz}$ which can be observed both in the spectrum and the spectrograms. Furthermore, the comparison of the 
spectra reveals that the main contributions of the airfoil to the sound radiation are in the range between 100 and $900 \mathrm{~Hz}$. All in all, the aeroacoustics of the airfoil have a rather broad-band character with a more pronounced frequency component between 200 and $300 \mathrm{~Hz}$. Noteworthy is the peak around $740 \mathrm{~Hz}$ (marked with a dashed line in figure 4) in the case of the stationary airfoil, which is attributed to periodic vortex shedding from the trailing edge. This is supported by the fact that $740 \mathrm{~Hz}$ corresponds to a Strouhal number of 0.32 using the airfoil thickness at $80 \%$ chord as the characteristic length scale.

\subsection{Measured velocity fields}

In figure 6 , six out of the 14 available phase angles are selected for the discussion of the dynamic stall life cycle and its associated flow characteristics. Phase averages and standard deviations are calculated for each phase angle using approximately 1000 PIV snapshots. They are used to determine the cross-correlation values and the underlying velocity fluctuations. Figure 6 shows the phase averaged velocity fields for selected phase angles as a vector plot. Only every third velocity vector is plotted in each direction for the sake of visibility. The normalized vorticity is color-coded. For each phase angle, the angle of attack of the airfoil is indicated and an arrow indicates a pitching-up or pitching-down movement. The corresponding phase-locked standard deviations are presented in figure 7. They are a measure for the cycle-to-cycle velocity fluctuations. In addition, representative instantaneous velocity vector fields are shown in figure 8 for the six selected phase angles with the normalized vorticity color-coded. These serve to give a deeper insight into the prevailing flow behavior of the individual phase angles. 

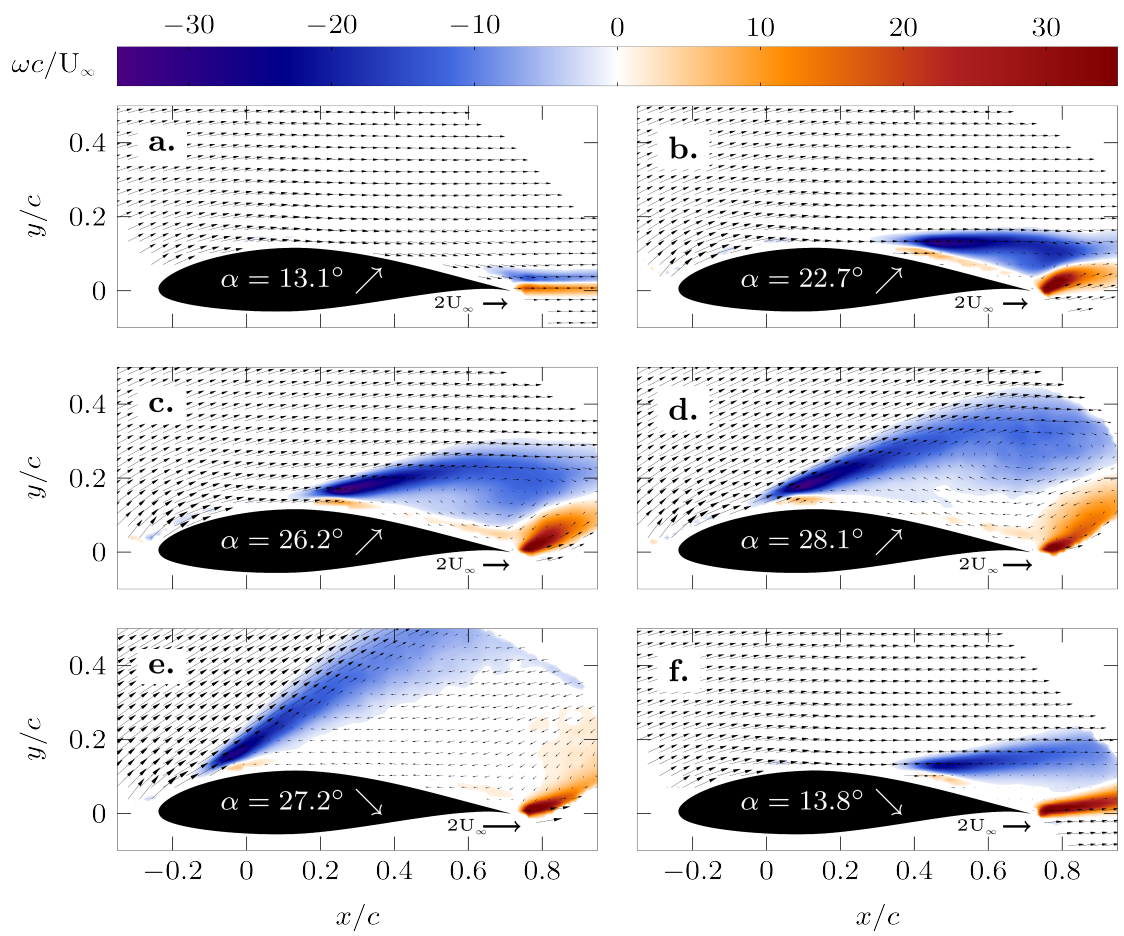

Fig. 6 Mean velocity vector fields for six selected phase angles representing the dynamic stall life cycle. The vorticity is color-coded.

The selected flow fields in figure 6 represent the various flow stages of the dynamic stall development according to Mulleners and Raffel (2013). The dynamic stall cycle starts at a moderate angle of attack $\left(\alpha=13.1^{\circ} \nearrow\right)$ with the attached flow stage. Here, the flow remains attached to the airfoils surface during the first part of the pitch-up motion of the airfoil (figure 8a). Near the leading edge, the velocity increases to approximately $1.5 U_{\infty}$. During the attached flow stage, velocity fluctuations occur only directly behind the trailing edge in a narrow, elongated wake (figure $6 \mathrm{a}$ and $8 \mathrm{a}$ ). The vorticity fields indicate a double shear layer in the wake with clockwise rotating vorticity coming from the suction side and anticlockwise rotating vorticity coming form the pressure side. The standard deviation 


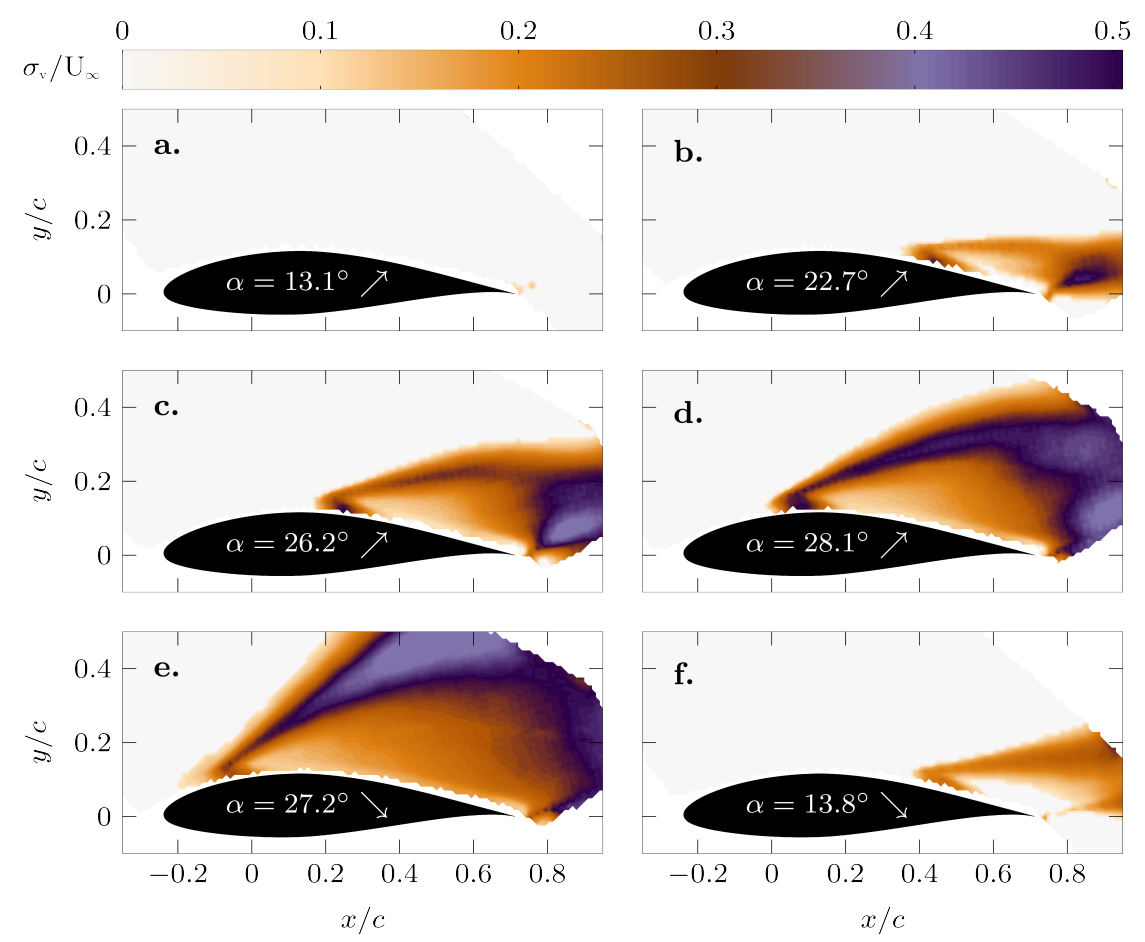

Fig. 7 Standard deviation of the velocity field for six selected phase angles representing the dynamic stall life cycle.

is very low overall, since the turbulent motions are very weak even in the wake region (figure 7a). This stage prevails for the first part of the upward motion.

With increasing angle of attack $\left(\alpha=22.7^{\circ} \nearrow\right)$ beyond the static stall angle of attack of approximately $18^{\circ}$ (Timmer, 2009), a negative vorticity region develops above the trailing edge indicating flow reversal (figure 6b). This is accompanied by significantly stronger flow fluctuations in a confined area behind the trailing edge (figure 7b). From this area, a shear layer is formed upstream (figure 8a) indicated by small-scale clockwise rotating vortices, which characterize the early stages of the dynamic stall. In addition, the velocity at the leading edge increases further to approximately twice the inflow velocity, which is associated with a higher lift 
coefficient.

The next stage $\left(\alpha=26.2^{\circ} \nearrow\right)$ is characterized by the expansion of the recirculation region and the movement of the separation point towards the leading edge (figure 6c). This advanced stall development is accompanied by the strongest fluctuation velocities in the recirculation area (figure 7c) and the highest velocity values at the leading edge in comparison with other stages. The most salient feature here is the clockwise rotating coherent structure within the reverse flow area (figure $8 \mathrm{c}$ ). Thereafter, the pitch-up motion ends and the separation point reaches its most upstream location marking the transition into a fully stalled stage (figure 6/8d-e). The shear layer is now significant enlarged and the recirculation region moves upwards and upstream with respect to the airfoil as it expands. Noteworthy is the formation of two counter-rotating vortical structures near $x / c=0.8$ in figure $7 / 8 \mathrm{~d}$ : a clockwise rotating structure underneath the shear layer and an opposed vortex in the detached flow near the trailing edge, which can be also observed in the instantaneous velocity field (figure 8d).

At the beginning of the downward motion of the airfoil $\left(\alpha=27.2^{\circ} \searrow\right)$ there is a noticeable drop in the maximum flow velocity at the leading edge of the airfoil, as well as a reduction in the maximum standard deviation due to the widestretched separation area (figure $7 \mathrm{e}$ ). The recirculation region has evolved into a fully developed turbulent wake flow (figure 8e), whereas the mean direction is still pointing against the inflow (figure 6e). The separation point is very close to the leading edge and the shear layer separates the inflow and the wake region in an almost straight line. The counter rotating vortices seem to prevail further downstream of the wake since the mean values show diverging directions of the velocity vectors in the upper and lower part of the wake. 


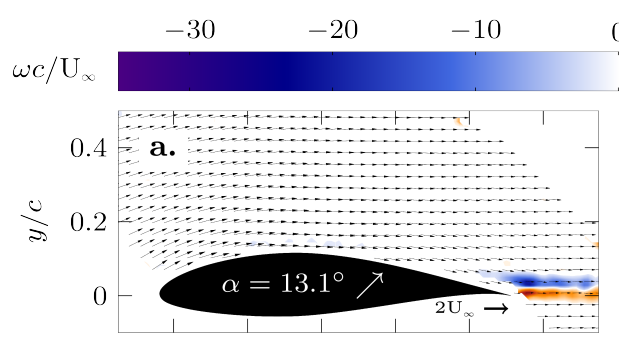

$\begin{array}{lll}0 & 20 & 30\end{array}$
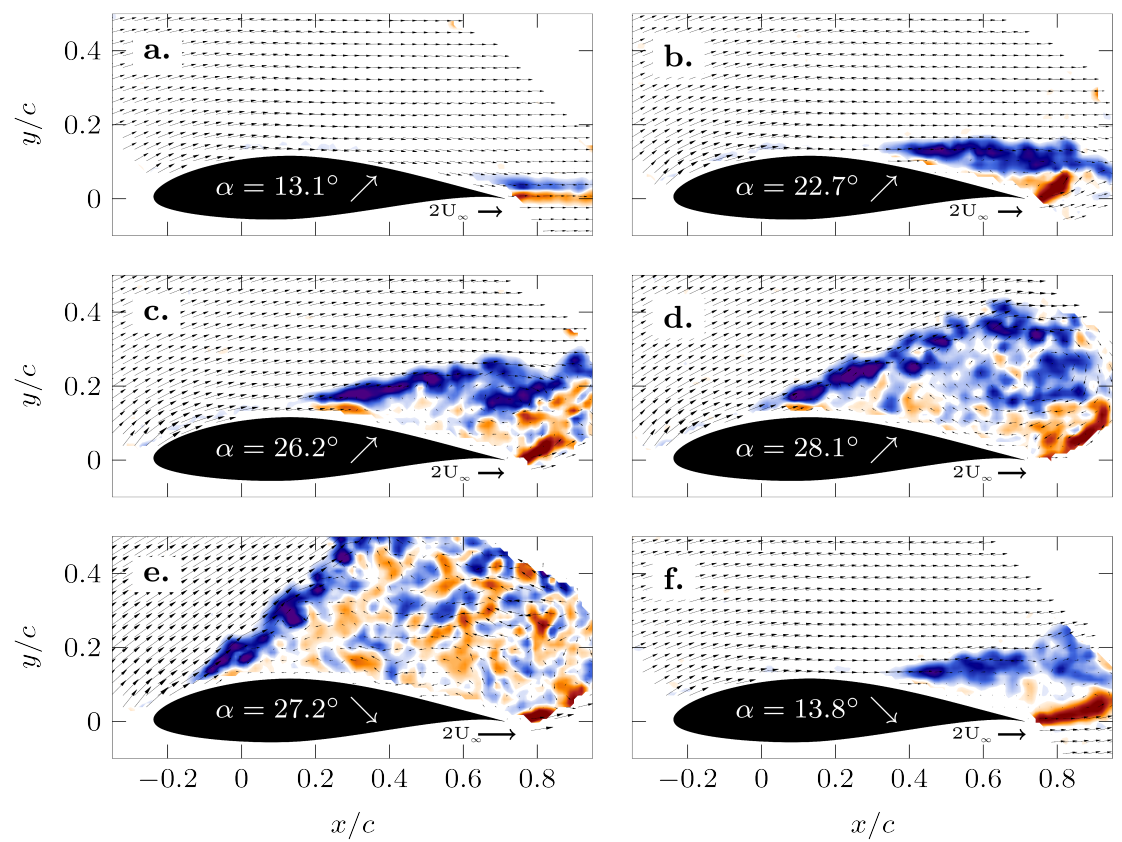

Fig. 8 Instantaneous velocity vector fields of six selected phase angles representing the dynamic stall life cycle. The vorticity is color-coded.

Lastly, the wake region settles again with further decreasing angle of attack $(\alpha=$ $13.8^{\circ} \nearrow$ ) indicating the flow reattachment. During this stage, the maximum flow velocity is the lowest in comparison to the flow situation during upstroke. It is noteworthy that the flow still shows increased fluctuations in the area close behind and above the trailing edge, especially in comparison to the equivalent angle of attack during the upstroke (figure $7 \mathrm{f}$ vs a). This is the so called hysteresis effect which is typical for the dynamic stall process. 


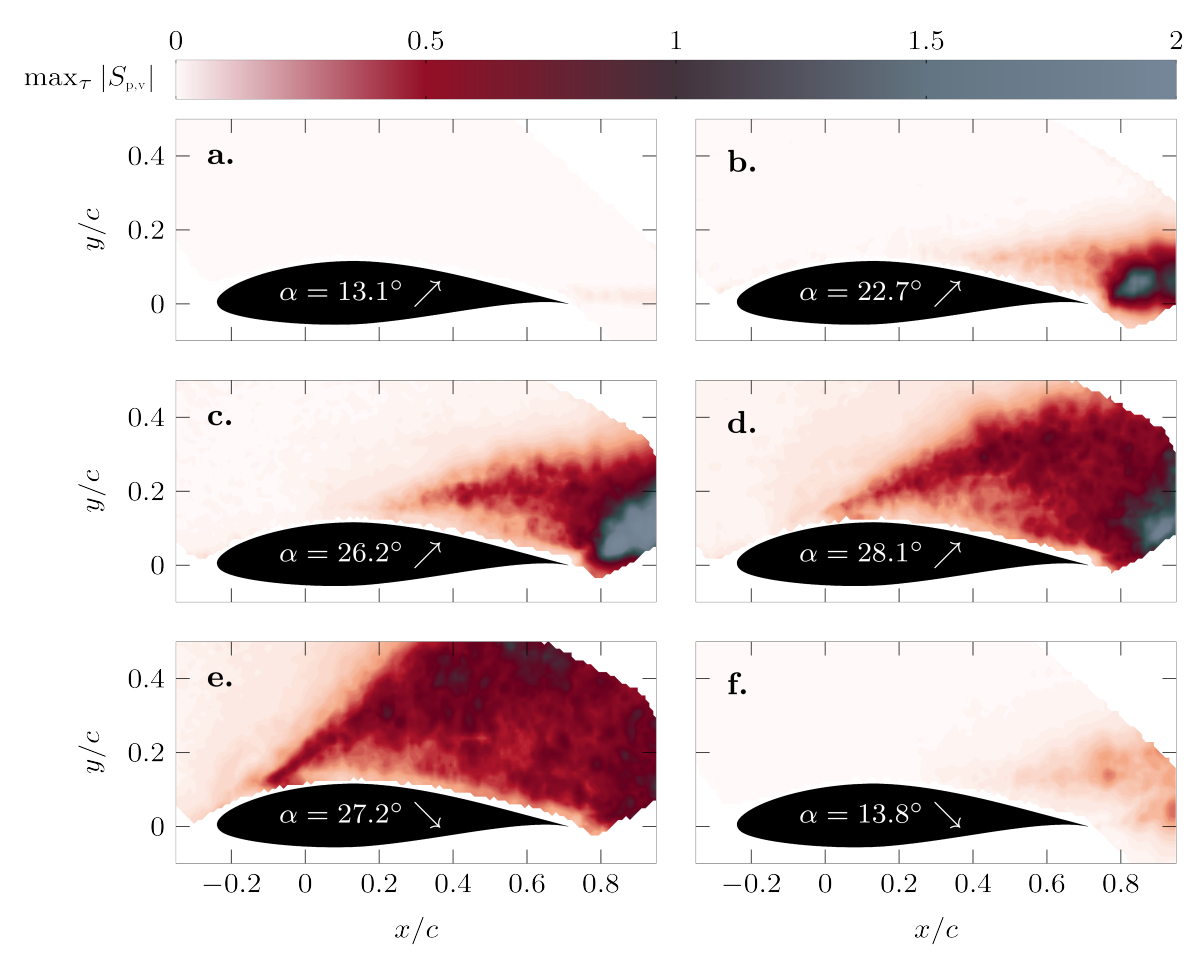

Fig. 9 Spatial distributions of the maxima with respect to $\tau$ of the absolute cross-correlation functions of the six selected phase angles representing the dynamic stall life cycle $(v$ component) based on ensembles of phases locked velocity snapshots.

4.3 Coherent structures represented by cross-correlation functions and coefficients

Different results of the cross correlation analysis are presented in order to demonstrate that the applicability and adequacy of the experimental approach to study the acoustic footprint of dynamic stall. Spatial distributions of the maxima of the absolute cross-correlation function $S_{p, v}$ and coefficients $R_{p, v}$ with respect to $\tau$ for the six selected phase angles based on the ensembles of phase-locked snaphots are shown in figure 9 and 10, respectively. This presentation provides an overview of where spatially coherent structures occur that are related to the sound emission in the statistical sense. The values of the $v$-velocity component are depicted, since 


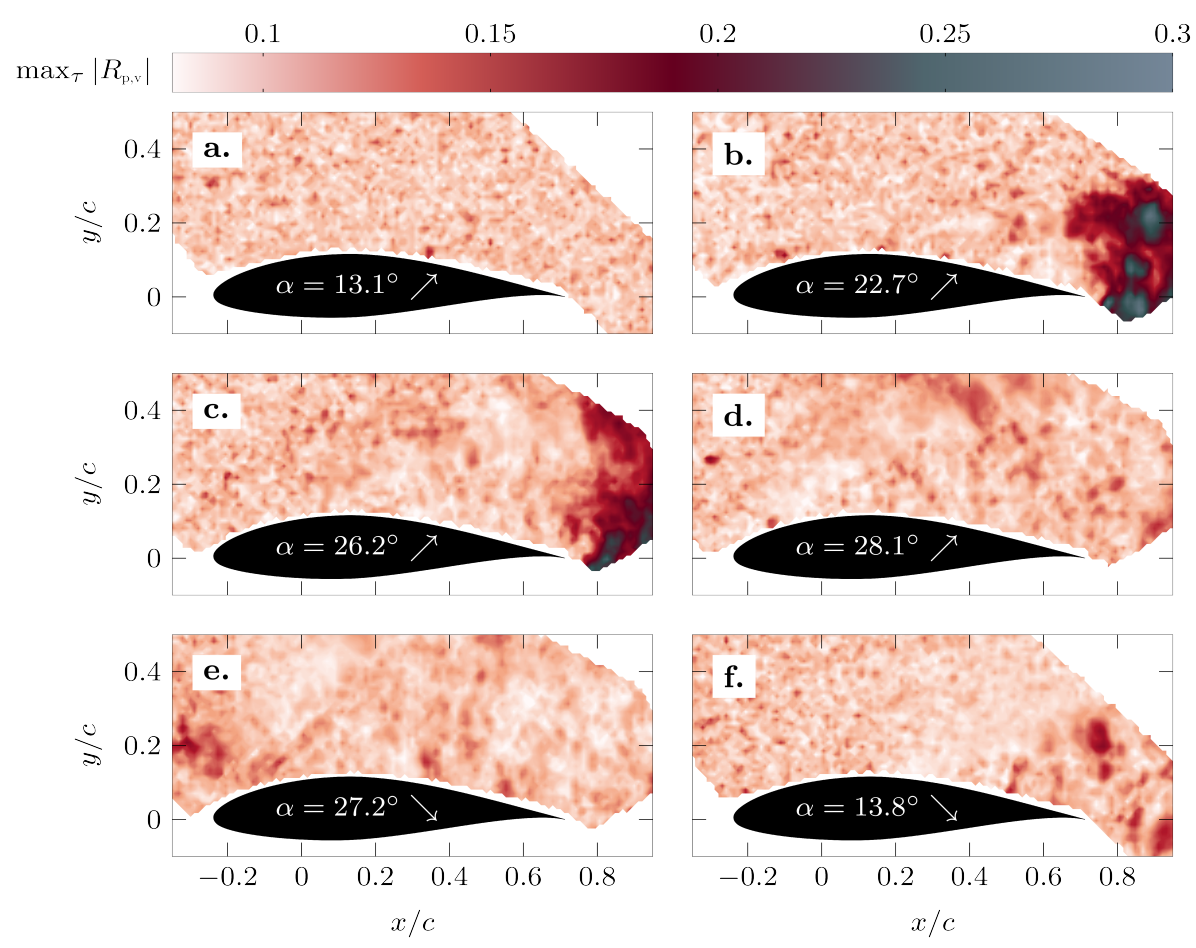

Fig. 10 Spatial distributions of the maxima with respect to $\tau$ of the absolute cross-correlation coefficients of the six selected phase angles representing the dynamic stall life cycle $(v$ component) based on ensembles of phases locked velocity snapshots.

they point in the direction in which the acoustic perturbations propagate to the microphones. In the case of the cross-correlation function, the values are scaled with the inflow velocity $U_{\infty}$ and the reference sound pressure $p_{0}$ in order to achieve a dimensionless representation.

Comparing the distributions of the cross-correlation function and the standard deviation values of the selected phase angles reveals that both analysis approaches lead to significant values in the same regions. Despite the fact that the distributions of the correlation functions appear somewhat blurred and stained, the main structures are still preserved. Therefore, the main features of the dynamic stall 
development are also represented on the basis of the correlation function. Since the cross-correlation operation provides a link between the acoustic and the velocity fluctuations, it is valid to conclude that regions with significant values are to a certain extent related to the noise source mechanisms. However, it is only with the help of the cross-correlation coefficients that a reliable statement about the quality of the correlation can be made because they specify the correlation with a percentage information. Hence, the coefficients are considered in combination with the airfoil self-noise mechanisms (Brooks et al, 1989) such as the vortex-shedding and the separation-stall noise to identify those flow structures associated with the noise emission at the respective phase angles.

At first glance it becomes clear that the presence of coherent structures that contribute to the sound emission is highly dependent on the phase angle of the airfoil's motion. Prominent acoustically relevant structures occur mainly during the stall development stages where flow reversal spreads over the airfoil chord. It can be assumed that at these phase angles periodic vortex shedding is present. This observation is supported by the temporal evolution of the coefficients at the points where the global maximum of the absolute value occur (figure 11). This figure also shows the error margins of the cross-correlation coefficients based on a t-test against zero with $99 \%$ probability, which are approximately \pm 0.08 for the $\approx 1000$ PIV snapshots used here per phase angle. Consequently, it can be stated that the detected periodic vortex shedding is most likely not a coincidence, as the values clearly exceed these error limits in the strongly periodic range with a frequency of $\approx 245 \mathrm{~Hz}$ and $\approx 220 \mathrm{~Hz}$ in case (b) and (c), respectively. In contrast, the correlation coefficient results during the attached flow do not reveal acoustically relevant flow structure (figure 10) and the values of the temporal evolution 

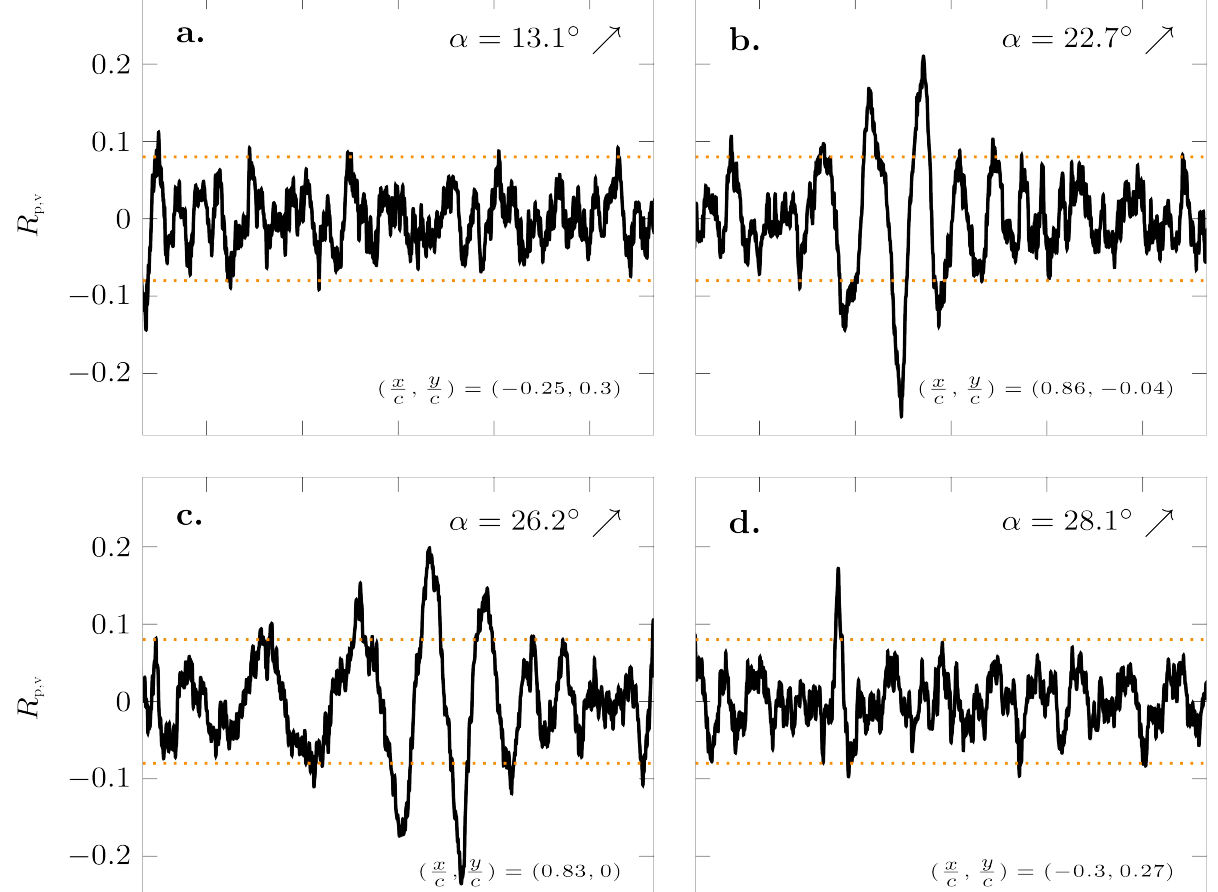

d. $\quad \alpha=28.1^{\circ} \nearrow$
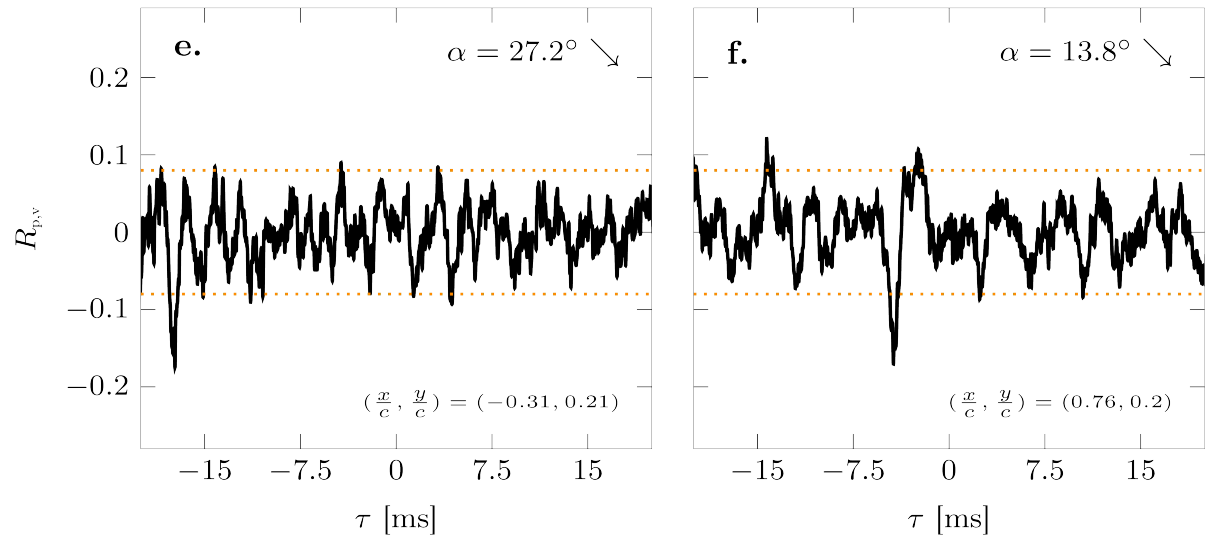

Fig. 11 Temporal evolution of the cross-correlation coefficients at the points where the global maximum of the absolute values occurs for the six selected phase angles representing the dynamic stall life cycle ( $v$-component) based on ensembles of phases locked velocity snapshots. 
of $R_{p, v}$ remain within the error bands. This is not surprising as the flow follows the airfoils contour nicely and only a very thin wake region is observed in which noise-generating vortical structure can develop. Based on the low values of the cross correlation function and the standard deviation, it can be concluded that there are no noise-generating flow effects in this phase angle range. In addition, that the overall sound pressure level for the lower angle of attack ranges is low.

Even during full stall (d-e) there are no clear coherent structures that can be identified in the spatial distribution of the maximum of the correlation coefficient with respect to $\tau$, even though the corresponding distribution of the crosscorrelation function and standard deviation are much higher here than during attached flow. In the temporal evolution, the signal remains within the error bands.

When the separation region is large in (d) and (e) there are also no distinct coherent structures observable in the spatial distributions of the maxima in relation to $\tau$ of the absolute cross-correlation coefficients even though the corresponding cross-correlation function and standard deviation values are much higher than in stage (a). While the flow development during the growth of the separation region is dominated by smaller scale structures that can be identified within the field of view, the flow during full stall is dominated by larger scale structures that cause lower frequency responses in the fluctuations. The latter are much harder to identify in the cross-correlation results. In the temporal evolution, only a short, conspicuous event occurs with a significant portion above the error margin.

During reattachment, the flow structures are again smaller and identifiable in confined areas within the region of flow separation. However, the coherent motion is still superimposed with random turbulent fluctuations resulting in a less pro- 

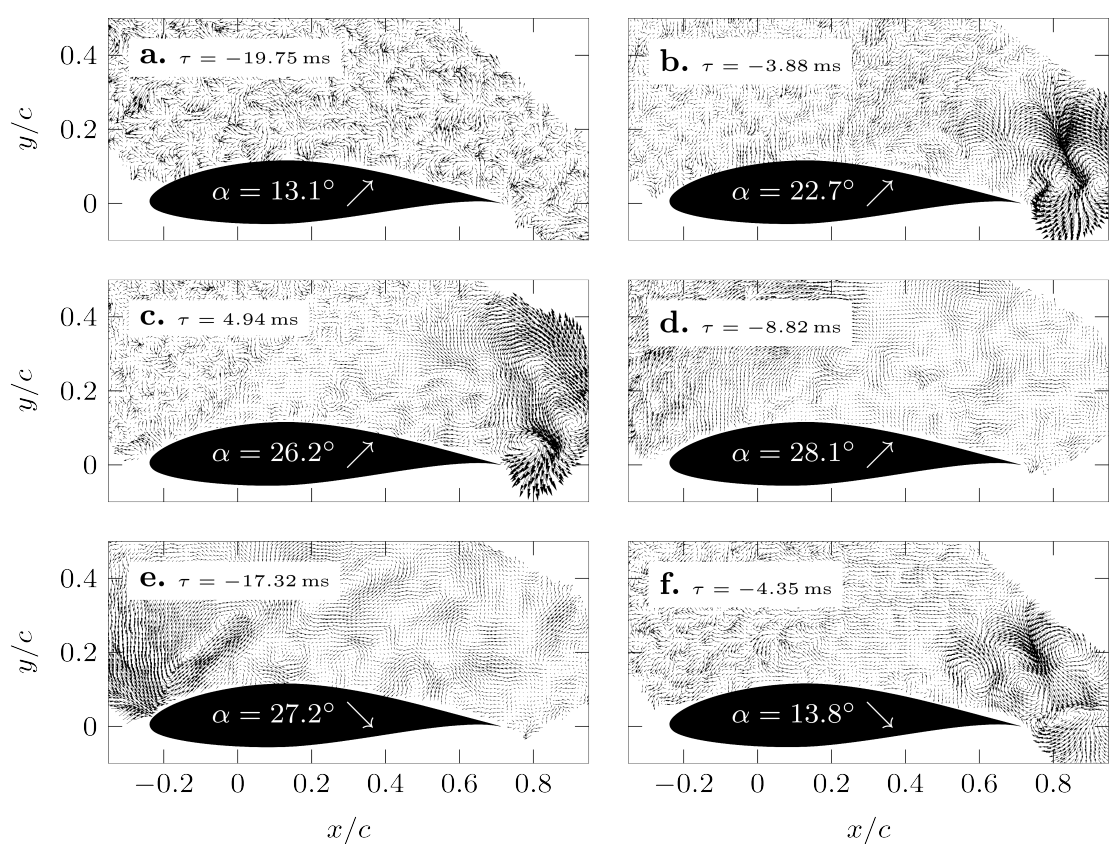

Fig. 12 Instantaneous vector fields of the cross-correlations coefficients based on ensembles of phases locked velocity snapshots at the points in time when the absolute values are maximal for six selected phase angles.

nounced periodic shedding in comparison with the separation region growth stages (figure $11 \mathrm{f}$ vs b-c). The inherent hysteresis associated with dynamic stall is once again evident from the direct comparison of the results at an angle of attack of 13 during pitch-up and during pitch-down, respectively.

In order to gain a further insight into the characteristic structures during dynamic stall development at the individual phase angles, the instantaneous vector fields of the cross-correlation coefficients at the point in time where the values are maximal are studied (figure 12). In figure 12, all available vectors are plotted to obtain a better overview of the various structures. As expected, during attached flow no structures are identified (figure 12a) but the periodic vortex shedding during the growth of the separation region is nicely visualized (figure 12b-c). In figure $12 \mathrm{~b}$, 
two counter-rotating vortical structures are identified in the wake behind the airfoils trailing edge. As indicated by the corresponding time evolution in figure 11b, these structures are convected downstream in a periodic manner with a frequency of $\approx 245 \mathrm{~Hz}$ corresponding to a Strouhal number of 0.245 based on the height of the separation region at the trailing edge. The case (c) shows a subsequent stage of the vortex shedding where the trajectory of the vortex shedding is shifted upwards in the airfoils frame of reference. This is due to the increased angle of attack. Additionally, the distance between the counter-rotating structures is slightly increased but the periodicity is preserved with a frequency of $\approx 220 \mathrm{~Hz}$ corresponding to a Strouhal number of 0.411 based on the increased height of the separation region at the trailing edge. Furthermore, small-scaled turbulent structures can now be detected within the entire separation region. When approaching full stall, the number of small scale structures within the separation region increases reflecting the turbulent character of the flow behavior. The large turbulent separated flow region stretches diagonally above the wing downstream. At maximum angle of attack a relative clear separation between the accelerated inflow and the turbulent region can be noticed while a more fluent transition is observed in the beginning of the downstroke motion. In the latter case, the correlation field near the leading edge is enhanced and this effect spreads along the shear layer edge. All in all, both cases exhibit a characteristic behavior that can be attributed to the separationstall noise (Brooks et al, 1989), which is characterized by the noise radiation from the chord as a whole.

During flow reattachment, counter-rotating vortical structures emerge again (figure 12f) but the temporal evolution of the coefficient does not allow for the identification of a distinct shedding frequency. 


\section{Conclusion}

The aeroacoustic relationship between flow structures that are generated by an oscillating airfoil and the acoustic radiation in the far field is investigated by means of simultaneous PIV and microphone measurements. In the acoustic spectrum a maximum amplitude around $750 \mathrm{~Hz}$ is attributed to the periodic vortex separation at the trailing edge of the airfoil. Further maxima at lower frequencies in the range of $250 \mathrm{~Hz}$ correspond to the large scale vortex shedding occurring during the onset of the stall.

Selected flow fields at six angles of attack during the up- and downstroke of the airfoil pitching cycle that represent different stages of the dynamic stall flow development were analyzed by means of a causality correlation method. For the selected phase angles, the phase locked PIV velocity fluctuations on the suction side of the airfoil were cross-correlated with the pressure fluctuations recorded with microphones in the acoustic far-field. By means of the causality correlation, acoustic signals can be associated with the coherent flow patterns that cause them. The presence of coherent structures that contribute to the sound emission is highly dependent on the phase angle of the airfoil's motion. In the beginning of the upstroke, when the flow is attached, no noise sources are identified in the flow field. With increasing angle of attack, vortices start shedding in the wake of the airfoil and give rise to significant correlation coefficients and noise. The Strouhal numbers of the vortex shedding at different phase angles we determined between 0.25 and 0.41 based on the temporal evolution of the cross-correlation coefficients. When the flow is fully stalled, the region with significant cross-correlation coefficients increases while the maximum values within the field of view decrease. This is due to 
the fact that the locations of the coherent structures that contribute to the sound emission during full stall fluctuate stronger between oscillation cycles than during stall development and even reattachment. The sound pressure levels however reach the highest values during full stall. The cycle-to-cycle variations of vortex generation and shedding during full stall thus smear the causality correlation coefficients based on phase-locked data across the entire chord length and do not allow for individual noisy generating structures to be identified. During stall development and flow reattachment, the phase-locked data is well suited to identify the wake structures causing noise. Furthermore, hysteresis is clearly observed on the flow topology at similar angles of attack during up and downstroke and in the acoustic signature.

\section{References}

Bossanyi EA (2003) Individual blade pitch control for load reduction. Wind Energy 6:119-128

Brooks TF, Humphreys Jr WM (2003) Flap-edge aeroacoustic measurements and predictions. Journal of Sound and Vibration 261:31-74

Brooks TF, Pope DS, Marcolini MA (1989) Airfoil self-noise and prediction. NASA Reference Publication 1218 p 142

Carr LW (1988) Progress in analysis and prediction of dynamic stall. AIAA Journal 25(1):6-17

Carr LW, McAlister KW, McCroskey WJ (1977) Analysis of the delopment of dynamic stall based on oscillating airfoil experiments. Technical Report, NASA 
Degani AT, Walker JDA, T SF (1998) Unsteady separation past moving surfaces. Journal of Fluid Mechanics 375:1-38

Doligalski TL, Smith CR, Walker JDA (1994) Vortex interactions with walls. Annu Rev Fluid Mech 26:573-616

Doolan CJ, Moreau DJ, Brooks LA (2012) Wind turbine noise mechanisms and some concepts for its control. Acoustics Australia 40(1):7-13

Ffowcs Williams JE, Hawkings DL (1969) Sound Generation by Turbulence and Surfaces in Arbitrary Motion. Philosophical Transactions of the Royal Society of London Serie A, Mathematical and Physical Sciences 264(1151):321-342

Gupta R, Ansell PJ (2017) Unsteady flow physics of airfoil dynamic stall. In: 55th AIAA Aerospace Sciences Meeting, Reston, Virginia

Henning A, Koop L, Ehrenfried K (2010) Simultaneous Particle Image Velocimetry and Microphone Array Measurements on a Rod-Airfoil Configuration. AIAA Journal 48(10):2263-2273

Henning A, Wrede B, Geisler R (2012) Aeroacoustic Investigation of a High-Lift Device by Means of Synchronized PIV and Microphone Measurements. In: 16th Int Symp on Applications of Laser Techniques to Fluid Mechanics, Lisbon, Portugal

Manela A (2013) On the acoustic radiation of a pitching airfoil. Physics of Fluids $25: 16$

McAlister KW, Carr LW, McCroskey WJ (1978) Dynamic stall experiments on the NACA 0012 airfoil. Technical Report, NASA

McCroskey WJ (1981) The Penomenon of Dynamic Stall. Technical Report, NASA

Mulleners K, Raffel M (2012) The onset of dynamic stall revisited. Experiments in Fluids 52:779-793 
Mulleners K, Raffel M (2013) Dynamic stall development. Experiments in Fluids 54:1469-1477

Nagarajan S, Hahn S, Lele SK (2006) Prediction of Sound Generated by a Pitching Airfoil: A Comparison of RANS and LES. In: 12th AIAA/CEAS Aeroacoustics Conference, Cambridge, Massachusetts

Obabko AV, Cassel KW (2002) Detachment of the Dynamic-Stall Vortex Above a Moving Surface. AIAA Journal 40(9):1811-1822

Oerlemans S, Migliore P (2004) Aeroacoustic wind tunnel tests of wind turbine airfoils. 10th AIAA/CEAS Aeroacoustics Conference; Manchester, United Kingdom

Petrovic V (2008) Reduction of wind turbine structural loads based on individual pitch control. Annual Conference of IEEE Industrial Electronics

Raffel M, Kompenhans J, Wernert P (1995) Investigation of the unsteady flow velocity field above an airfoil pitching under deep dynamic stall conditions. Experiments in Fluids 19:103-111

Raffel M, Willert CE, Wereley ST, Kompenhans J (2007) Particle Image Velocimetry. Springer Berlin Heidelberg

Reynolds WC, Carr LW (1992) Review of unsteady, driven separated flows. Technical Report, AIAA

Timmer WA (2009) An overview of NACA 6-digit airfoil series characteristics with reference to airfoils for large wind turbine blades. In: 47th AIAA Aerospace Sciences Meeting including The New Horizons Forum and Aerospace Exposition, AIAA 2009-268

Wolf A, Lutz T, Würz W, Krämer E, Stalnov O, Seifert A (2014) Trailing edge noise reduction of wind turbine blades by active flow control. Wind Energy 
$17: 657-669$

Zanotti A, Ermacora M, Campanardi G, Gibertini G (2014) Stereo particle image velocimetry measurements of perpendicular blade-vortex interaction over an oscillating airfoil. Experiments in Fluids 55:1811 